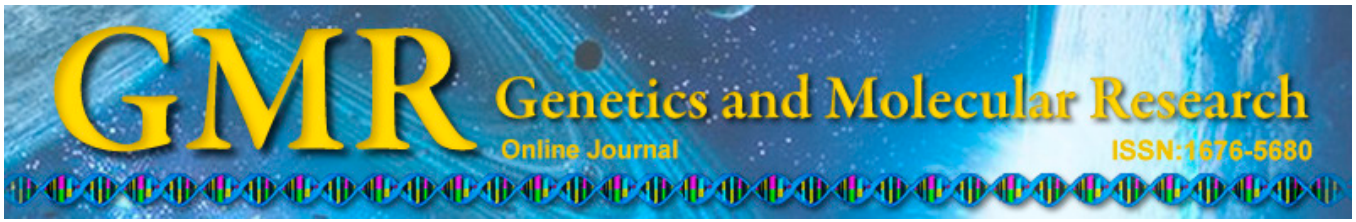

\title{
Phylogenetic analysis of Gansu sheeppox virus isolates based on P32, GPCR, and RPO30 genes
}

\author{
H.L. Su${ }^{1}$, H.J. Jia ${ }^{2}$, C. Yin ${ }^{2}$, Z.Z. Jing ${ }^{2}$, X.N. Luo ${ }^{2}$ and Y.X. Chen ${ }^{1}$ \\ ${ }^{1}$ College of Life Science and Engineering, Northwest University for Nationalities, \\ Lanzhou, China \\ ${ }^{2}$ State Key Laboratory of Veterinary Etiological Biology, \\ Key Laboratory of Veterinary Parasitology of Gansu Province, \\ Lanzhou Veterinary Research Institute, CAAS, Lanzhou, China
}

Corresponding authors: Y.X. Chen / X.N. Luo

E-mail: chenyx69@126.com / luoxuenong@caas.cn

Genet. Mol. Res. 14 (1): 1887-1898 (2015)

Received February 27, 2014

Accepted October 7, 2014

Published March 13, 2015

DOI http://dx.doi.org/10.4238/2015.March.13.17

\begin{abstract}
Two outbreaks of sheeppox in sheep have occurred in Gansu Province, China. The P32, GPCR, and RPO30 genes were used as markers for differential diagnosis. We confirmed that the outbreaks were caused by sheeppox virus. Sequence and phylogenetic analysis of the P32, GPCR, and RPO30 genes revealed a close relationship between the 2 isolates and Chinese sheeppox viruses. Because ill sheep were imported from Jingyuan, another county of Gansu Province, our results strongly suggest the importance of veterinary surveillance prior to transportation.
\end{abstract}

Key words: $G P C R ; P 32 ; R P O 30$; Sheeppox virus 


\section{INTRODUCTION}

Sheeppox virus (SPPV) is a member of the genus Capripoxvirus of the family Poxviridae, which also contains 2 other members, goatpox virus (GTPV) and lumpy skin disease virus (LSDV) (Fields et al., 2007). SPPV can infect both sheep and goats and has been reported in many areas in the world, including Asia, Europe, Africa, America, and Oceania (Asagba and Nawathe, 1981; Afshar et al., 1986; Oğuzoğlu et al., 2006; Roy et al., 2008; Beard et al., 2010; Bhanuprakash et al., 2006, 2010). Infections by SPPV can cause high morbidity and mortality, particularly in lambs (Rao and Bandyopadhyay, 2000), subjecting sheepherders to considerable economic losses. Because of its great impacts on animal health, sheeppox is categorized as a notifiable disease by the World Organization for Animal Health.

Clinically, animals infected by SPPV and GTPV exhibit a fever with the development of macules in the skin, making primary diagnosis easy; however, differential diagnosis is difficult (Bhanuprakash et al., 2006; Gulbahar et al., 2006; Oğuzoğlu et al., 2006; Embury-Hyatt et al., 2012). Therefore, several molecular markers, including P32, G-protein-coupled receptors $(G P C R)$, and RNA polymerase subunit (RPO30), have been proposed for use in differential diagnosis. $P 32$ is an envelope protein of the capripoxvirus and is homologous to the $P 35$ protein encoded by the vaccinia virus $H 3 L$ gene (Johnson et al., 1993; Heine et al., 1999). Because of the size difference of $P 32$ between SPPV and GTPV, they can be distinguished by sequence comparison (Tian et al., 2010). GPCR, which is involved in defense against the aggressive assault executed by host inflammatory responses (Chensue, 2001; Seet and McFadden, 2002), has been used for differential diagnosis of SPPV, GTPV, and LSDV (Le Goff et al., 2009; Lamien et al., 2011b). The RPO30 gene, a homolog of the vaccinia virus $E 4 L$ gene, encodes a 30-kDa DNA-dependent RNA polymerase subunit (Ahn et al., 1990; Tulman et al., 2002). Although the RPO30 gene is conserved among capripoxviruses, it is still used to distinguish between SPPV, GTPV, and LSDV (Lamien et al., 2011a; Zhou et al., 2012).

In this study, we report 2 cases of SPPV in sheep in Gansu Province, China. We used $P 32, G P C R$, and RPO30 as molecular biomarkers for phylogenetic analysis.

\section{MATERIAL AND METHODS}

\section{Treatment of samples}

Papules or scabs were collected from ill sheep in the Zhangye and Huining counties of Gansu Province, China. In both cases, macules were observed on the lips, udders, and tails. Papules or scabs were first suspended in $0.01 \mathrm{M}$ phosphate-buffered saline, $\mathrm{pH}$ 7.4. Next, the samples were homogenized in the lab for later use.

\section{Propagation in cell culture}

The homogenized samples were filtered using a $0.45-\mu \mathrm{m}$ filter (Millipore, Billerica, MA, USA). Next, $2 \mathrm{~mL}$ filtrate was inoculated into lamb testis cell culture for virus isolation. When a cytopathic effect occurred, the cells were harvested 6 days post-inoculation by 3 cycles of alternating freezing and thawing.

\section{Extraction of viral genomic DNA}

After the lysate was centrifuged at $664 \mathrm{~g}$ for $10 \mathrm{~min}$, the DNA was extracted according 
to the instructions of the Axygen ${ }^{\mathrm{TM}}$ Viral DNA/RNA Miniprep kit (Axygen, Union City, CA, USA). The extracted DNA was stored at $-20^{\circ} \mathrm{C}$.

\section{Amplification of P32, GPCR, and RPO30}

The following specific primers for $P 32, G P C R$, and $R P O 30$ genes were designed using primer 5.0 and synthesized by TaKaRa (Dalian, China):

P32-F: 5'-ATG GCA GAT ATC CCA TT-3'; P32-R: 5'-CTA AAC TAT ATA CGT AAA TAA CAT AC-3'; GPCR-F: 5'-TTT ATC AGC ACT AGG TCA TTA TCT-3'; GPCR-R: 5'-TAT CAC TCC CTT CCA TTT TTA T-3'; RPO30-F: 5'-CTC TGT TCC AAA CTA AAT CAT-3'; RPO30-R: 5'-TTT TTG TAT TAC CAA TTT CTG-3'; The polymerase chain reaction (PCR) system ( $50 \mu \mathrm{L}$ total volume) included $1 \mu \mathrm{L}$ extracted DNA, $5 \mu \mathrm{L}$ 10X PCR buffer, $0.3 \mu \mathrm{L}$ LA Taq DNA polymerase (5 U/ $\mu \mathrm{L}$ ) (TaKaRa), $4 \mu \mathrm{L} 2.5 \mathrm{mM}$ dNTPs, $38.2 \mu \mathrm{L}$ nuclease-free water, and $0.75 \mu \mathrm{L}$ of each primer $(10 \mu \mathrm{M})$, and was performed in a Personal Thermal Cycler (Bio-Rad, Hercules, CA, USA) with the following conditions: denaturation for $4 \mathrm{~min}$ at $98^{\circ} \mathrm{C}$, then 35 cycles of denaturation for $30 \mathrm{~s}$ at $95^{\circ} \mathrm{C}$, annealing for $30 \mathrm{~s}$ at $52^{\circ} \mathrm{C}$ for $P 32,49.5^{\circ} \mathrm{C}$ for $G P C R$, or $53^{\circ} \mathrm{C}$ for $R P O 30$, extension for $120 \mathrm{~s}$ at $72^{\circ} \mathrm{C}$, and a final elongation for $10 \mathrm{~min}$ at $72^{\circ} \mathrm{C}$. The products were examined by $1 \%$ agarose gel electrophoresis with ethidium bromide staining. The products were then purified using the AxyPrep ${ }^{\mathrm{TM}}$ DNA Gel Extraction kit (Axygen). The samples were sequenced.

\section{Phylogenetic analysis}

The nucleotide sequences of the $P 32, G P C R$, and RPO30 genes of other capripoxvirus strains were retrieved from GenBank (Table 1). ClustalW was used to align these sequences. Phylogenetic analysis was performed using neighbor-joining, maximum parsimony, maximum-likelihood, and minimum evolution in MEGA 5.10 (McCormack and Clewley, 2002; Chakraborty et al., 2013). The topology of the trees was tested by bootstrap analysis with 1000 replicates (Sanderson and Wojciechowski, 2000).

\section{RESULTS AND DISCUSSION}

In both cases, macules were observed on the lips, udders, and tails (Figure 1). Compared with other capripoxvirus isolates, the Huining and Zhangye isolates shared 97.9-99.9\% nucleotide identity and 72.3-99.7\% amino acid identity for $P 32$ (Table 2), 95.0-99.7 and 93.0$100 \%$ for GPCR (Table 3), and $96.2-100$ and $97.4-100 \%$ for $R P O 30$, respectively (Table 4). Based on the results of amino acid sequence comparison of P32, GPCR, and RPO30, the 2 isolates showed no unique amino acid substitutions compared with other members. The alignment of the partial amino acid sequences of the P32 genes showed that our strains (HN and ZYGT) had the highest similarity to other SPPV and LSDV strains (Figure 2). Moreover, the putative amino acid sequences of Huining and Zhangye isolates contained an aspartic acid at the 55th position, which is absent in GTPV (Hosamani et al., 2004). Combined with the alignment results for GPCR and RPO30 (data not shown), the 2 isolates were SPPV. Similar topological trees were obtained using the 3 genes above (Figures 3-5). In the phylogenetic trees based on the $P 32, G P C R$, and $R P O 30$ sequences, the viruses were clearly segregated into 3 major groups: SPPV, GTPV, and LSDV. The 2 isolates were clustered with the SPPV branch, and clustered together with the Asia isolates. 
Table 1. Capripoxvirus strains used for phylogenetic analysis.

\begin{tabular}{|c|c|c|c|c|c|c|}
\hline Gene & Virus designation & Host & $\begin{array}{l}\text { Accession } \\
\text { No. }\end{array}$ & $\begin{array}{l}\text { Species of } \\
\text { capripoxvirus }\end{array}$ & $\begin{array}{l}\text { Country of } \\
\text { isolation }\end{array}$ & Reference \\
\hline P32 & Huining isolate & Sheep & & SPPV & Huining (China) & This study \\
\hline P32 & Zhangye isolate & Sheep & & SPPV & Zhangye (China) & This study \\
\hline P32 & China-EF522177.1-GTPV & Goat & EF522177.1 & GTPV & China & Unpublished \\
\hline P32 & China-EF522180.1-GTPV & Goat & EF522180.1 & GTPV & China & Unpublished \\
\hline P32 & China-EF522176.1-GTPV & Goat & EF522176.1 & GTPV & China & Unpublished \\
\hline P32 & Vietnam-EU625263.1-GTPV & Goat & EU625263.1 & GTPV & Vietnam & (Babiuk et al., 2009) \\
\hline P32 & China-HM572329.1-GTPV & Goat & HM572329.1 & GTPV & China & Unpublished \\
\hline P32 & China-EF514890.1-GTPV & Goat & EF514890.1 & GTPV & China & Unpublished \\
\hline P32 & China-AY773088.1-GTPV & Goat & AY773088.1 & GTPV & China & Unpublished \\
\hline P32 & China-EF514892.1-GTPV & Goat & EF514892.1 & GTPV & China & Unpublished \\
\hline P32 & India-FJ748488.1-GTPV & Goat & FJ748488.1 & GTPV & India & Unpublished \\
\hline P32 & China-JN602370.1-GTPV & Goat & JN602370.1 & GTPV & China & (Zhou et al., 2012) \\
\hline P32 & India-AY159333.1-GTPV & Goat & AY159333.1 & GTPV & India & (Hosamani et al., 2004) \\
\hline P32 & China-AY881707.1-GTPV & Goat & AY881707.1 & GTPV & China & NA \\
\hline P32 & India-AY382869.1-GTPV & Goat & AY382869.1 & GTPV & India & (Hosamani et al., 2004) \\
\hline P32 & China-EF514889.1-GTPV & Goat & EF514889.1 & GTPV & China & Unpublished \\
\hline P32 & China-EF522178.1-GTPV & Goat & EF522178.1 & GTPV & China & Unpublished \\
\hline P32 & China-JN596275.1-GTPV & Goat & JN596275.1 & GTPV & China & (Zhou et al., 2012) \\
\hline P32 & China-EF514891.1-GTPV & Goat & EF514891.1 & GTPV & China & Unpublished \\
\hline P32 & Yemen-EU625262.1-GTPV & Goat & EU625262.1 & GTPV & Yemen & (Babiuk et al., 2009) \\
\hline P32 & India-DQ153219.1-SPPV & Sheep & DQ153219.1 & SPPV & India & Unpublished \\
\hline P32 & India-DQ153224.1-SPPV & Sheep & DQ153224.1 & SPPV & India & Unpublished \\
\hline P32 & India-DQ153223.1-SPPV & Sheep & DQ153223.1 & SPPV & India & Unpublished \\
\hline P32 & India-DQ153225.1-SPPV & Sheep & DQ153225.1 & SPPV & India & Unpublished \\
\hline P32 & India-DQ153220.1-SPPV & Sheep & DQ153220.1 & SPPV & India & Unpublished \\
\hline P32 & India-DQ153221.1-SPPV & Sheep & DQ153221.1 & SPPV & India & Unpublished \\
\hline P32 & India-DQ153226.1-SPPV & Sheep & DQ153226.1 & SPPV & India & Unpublished \\
\hline P32 & India-AY368684.1-SPPV & Sheep & AY368684.1 & SPPV & India & Unpublished \\
\hline P32 & China-JN596274.1-SPPV & Sheep & JN596274.1 & SPPV & China & (Zhou et al., 2012) \\
\hline P32 & India-FJ882029.1-SPPV & Goat & FJ882029.1 & SPPV & India & Unpublished \\
\hline P32 & India-FJ748487.1-SPPV & Goat & FJ748487.1 & SPPV & India & Unpublished \\
\hline P32 & China-HQ607368.1-SPPV & Sheep & HQ607368.1 & SPPV & China & NA \\
\hline P32 & South Africa-AF409137.1-LSDV & Cattle & AF409137.1 & LSDV & South Africa & (Kara et al., 2003) \\
\hline P32 & Kenya-AF325528.1-LSDV & Cattle & AF325528.1 & LSDV & Kenya & (Tulman et al., 2001) \\
\hline P32 & Kenya-NC003027.1-LSDV & Cattle & NC003027.1 & LSDV & Kenya & (Tulman et al., 2001) \\
\hline GPCR & Huining isolate & Sheep & & SPPV & Huining (China) & This study \\
\hline GPCR & Zhangye isolate & Sheep & & SPPV & Zhangye (China) & This study \\
\hline GPCR & China-JQ310667.1-GTPV & Goat & JQ310667.1 & GTPV & China & (Zhou et al., 2012) \\
\hline GPCR & Bangladesh-FJ869355.1-GTPV & Goat & FJ869355.1 & GTPV & Bangladesh & (Le Goff et al., 2009) \\
\hline GPCR & China-JQ310672.1-GTPV & Goat & JQ310672.1 & GTPV & China & (Zhou et al., 2012) \\
\hline GPCR & India-FJ869358.1-GTPV & Goat & FJ869358.1 & GTPV & India & (Le Goff et al., 2009) \\
\hline GPCR & Oman-FJ869359.1-GTPV & Goat & FJ869359.1 & GTPV & Oman & (Le Goff et al., 2009) \\
\hline GPCR & Chad-FJ869392.1-GTPV & Goat & FJ869392.1 & GTPV & Chad & (Le Goff et al., 2009) \\
\hline GPCR & Chad-FJ869363.1-GTPV & Goat & FJ869363.1 & GTPV & Chad & (Le Goff et al., 2009) \\
\hline GPCR & Burkina Faso-FJ869353.1-GTPV & Goat & FJ869353.1 & GTPV & Burkina Faso & (Le Goff et al., 2009) \\
\hline GPCR & Yemen-FJ869362.1-GTPV & Goat & FJ869362.1 & GTPV & Yemen & (Le Goff et al., 2009) \\
\hline GPCR & Turkey-FJ869356.1-GTPV & Goat & FJ869356.1 & GTPV & Turkey & (Le Goff et al., 2009) \\
\hline GPCR & Iraq-FJ869357.1-GTPV & Goat & FJ869357.1 & GTPV & Iraq & (Le Goff et al., 2009) \\
\hline GPCR & Sudan-FJ869369.1-LSDV & Cattle & FJ869369.1 & LSDV & Sudan & (Le Goff et al., 2009) \\
\hline GPCR & Egypt-FJ869377.1-LSDV & Cattle & FJ869377.1 & LSDV & Egypt & (Le Goff et al., 2009) \\
\hline GPCR & South Africa-FJ869375.1-LSDV & Cattle & FJ869375.1 & LSDV & South Africa & (Le Goff et al., 2009) \\
\hline GPCR & South Africa-FJ869373.1-LSDV & Cattle & FJ869373.1 & LSDV & South Africa & (Le Goff et al., 2009) \\
\hline GPCR & South Africa-FJ869372.1-LSDV & Cattle & FJ869372.1 & LSDV & South Africa & (Le Goff et al., 2009) \\
\hline$G P C R$ & Nigeria-FJ869368.1-LSDV & Cattle & FJ869368.1 & LSDV & Nigeria & (Le Goff et al., 2009) \\
\hline GPCR & Sudan-FJ869367.1-LSDV & Cattle & FJ869367.1 & LSDV & Sudan & (Le Goff et al., 2009) \\
\hline GPCR & Niger-FJ869366.1-LSDV & Cattle & FJ869366.1 & LSDV & Niger & (Le Goff et al., 2009) \\
\hline GPCR & Burkina Faso-FJ869352.1-LSDV & Cattle & FJ869352.1 & LSDV & Burkina Faso & (Le Goff et al., 2009) \\
\hline$G P C R$ & South Africa-FJ869374.1-LSDV & Cattle & FJ869374.1 & LSDV & South Africa & (Le Goff et al., 2009) \\
\hline GPCR & South Africa-FJ869371.1-LSDV & Cattle & FJ869371.1 & LSDV & South Africa & (Le Goff et al., 2009) \\
\hline GPCR & South Africa-FJ869370.1-LSDV & Cattle & FJ869370.1 & LSDV & South Africa & (Le Goff et al., 2009) \\
\hline$G P C R$ & Niger-FJ869365.1-LSDV & Cattle & FJ869365.1 & LSDV & Niger & (Le Goff et al., 2009) \\
\hline
\end{tabular}

Continued on next page 


\begin{tabular}{|c|c|c|c|c|c|c|}
\hline Gene & Virus designation & Host & $\begin{array}{l}\text { Accession } \\
\text { No. }\end{array}$ & $\begin{array}{l}\text { Species of } \\
\text { capripoxvirus }\end{array}$ & $\begin{array}{l}\text { Country of } \\
\text { isolation }\end{array}$ & Reference \\
\hline GPCR & China-JQ310675.1-SPPV & Sheep & JQ310675.1 & SPPV & China & (Zhou et al., 2012) \\
\hline GPCR & China-JQ310668.1-SPPV & Sheep & JQ310668.1 & SPPV & China & (Zhou et al., 2012) \\
\hline$G P C R$ & Turkey-FJ869389.1-SPPV & Sheep & FJ869389.1 & SPPV & Turkey & (Le Goff et al., 2009) \\
\hline GPCR & China-JQ310666.1-SPPV & Sheep & JQ310666.1 & SPPV & China & (Zhou et al., 2012) \\
\hline GPCR & Tunisia-FJ869347.1-SPPV & Sheep & FJ869347.1 & SPPV & Tunisia & (Le Goff et al., 2009) \\
\hline$G P C R$ & Tunisia-FJ869349.1-SPPV & Sheep & FJ869349.1 & SPPV & Tunisia & (Le Goff et al., 2009) \\
\hline GPCR & Morocco-FJ869378.1-SPPV & Sheep & FJ869378.1 & SPPV & Morocco & (Le Goff et al., 2009) \\
\hline GPCR & Niger-FJ869379.1-SPPV & Sheep & FJ869379.1 & SPPV & Niger & (Le Goff et al., 2009) \\
\hline GPCR & Tunisia-FJ869350.1-SPPV & Sheep & FJ869350.1 & SPPV & Tunisia & (Le Goff et al., 2009) \\
\hline$G P C R$ & Tunisia-FJ869351.1-SPPV & Sheep & FJ869351.1 & SPPV & Tunisia & (Le Goff et al., 2009) \\
\hline GPCR & Nigeria-FJ869381.1-SPPV & Sheep & FJ869381.1 & SPPV & Nigeria & (Le Goff et al., 2009) \\
\hline$G P C R$ & Nigeria-FJ869387.1-SPPV & Sheep & FJ869387.1 & SPPV & Nigeria & (Le Goff et al., 2009) \\
\hline GPCR & Algeria-FJ869385.1-SPPV & Sheep & FJ869385.1 & SPPV & Algeria & (Le Goff et al., 2009) \\
\hline GPCR & Algeria-FJ869386.1-SPPV & Sheep & FJ869386.1 & SPPV & Algeria & (Le Goff et al., 2009) \\
\hline GPCR & Tunisia-FJ869348.1-SPPV & Sheep & FJ869348.1 & SPPV & Tunisia & (Le Goff et al., 2009) \\
\hline$G P C R$ & Tunisia-FJ869346.1-SPPV & Sheep & FJ869346.1 & SPPV & Tunisia & (Le Goff et al., 2009) \\
\hline$G P C R$ & Tunisia-FJ869345.1-SPPV & Sheep & FJ869345.1 & SPPV & Tunisia & (Le Goff et al., 2009) \\
\hline GPCR & Turkey-FJ869388.1-SPPV & Sheep & FJ869388.1 & SPPV & Turkey & (Le Goff et al., 2009) \\
\hline$G P C R$ & Turkey-FJ869384.1-SPPV & Sheep & FJ869384.1 & SPPV & Turkey & (Le Goff et al., 2009) \\
\hline GPCR & Turkey-FJ869382.1-SPPV & Sheep & FJ869382.1 & SPPV & Turkey & (Le Goff et al., 2009) \\
\hline GPCR & Senegal-FJ869380.1-SPPV & Sheep & FJ869380.1 & SPPV & Senegal & (Le Goff et al., 2009) \\
\hline$G P C R$ & Turkey-FJ869383.1-SPPV & Sheep & FJ869383.1 & SPPV & Turkey & (Le Goff et al., 2009) \\
\hline RPO30 & Huining isolate & Sheep & & SPPV & Huining (China) & This study \\
\hline RPO30 & Zhangye isolate & Sheep & & SPPV & Zhangye (China) & This study \\
\hline RPO30 & Iraq-GU119942.1-GTPV & Goat & GU119942.1 & GTPV & Iraq & (Lamien et al., 2011a) \\
\hline RPO30 & Turkey-GU119940.1-GTPV & Goat & GU119940.1 & GTPV & Turkey & (Lamien et al., 2011a) \\
\hline RPO30 & Yemen-GU119927.1-GTPV & Goat & GU119927.1 & GTPV & Yemen & (Lamien et al., 2011a) \\
\hline RPO30 & Chad-GU119931.1-GTPV & Goat & GU119931.1 & GTPV & Chad & (Lamien et al., 2011a) \\
\hline RPO30 & Chad-GU119930.1-GTPV & Goat & GU119930.1 & GTPV & Chad & (Lamien et al., 2011a) \\
\hline RPO30 & Burkina Faso-GU119939.1-GTPV & Goat & GU119939.1 & GTPV & Burkina Faso & (Lamien et al., 2011a) \\
\hline RPO30 & Ghana-GU119935.1-GTPV & Goat & GU119935.1 & GTPV & Ghana & (Lamien et al., 2011a) \\
\hline RPO30 & China-JQ310674.1-GTPV & Goat & JQ310674.1 & GTPV & China & (Zhou et al., 2012) \\
\hline RPO30 & Bangladesh-GU119949.1-GTPV & Goat & GU119949.1 & GTPV & Bangladesh & (Lamien et al., 2011a) \\
\hline RPO30 & India-GU119936.1-GTPV & Goat & GU119936.1 & GTPV & India & (Lamien et al., 2011a) \\
\hline RPO30 & Oman-GU119933.1-GTPV & Goat & GU119933.1 & GTPV & Oman & (Lamien et al., 2011a) \\
\hline RPO30 & South Africa-GU119945.1-LSDV & Cattle & GU119945.1 & LSDV & South Africa & (Lamien et al., 2011a) \\
\hline RPO30 & Egypt-GU119947.1-LSDV & Cattle & GU119947.1 & LSDV & Egypt & (Lamien et al., 2011a) \\
\hline RPO30 & South Africa-GU119951.1-LSDV & Springbok & GU119951.1 & LSDV & South Africa & (Lamien et al., 2011a) \\
\hline RPO30 & South Africa-GU119950.1-LSDV & Cattle & GU119950.1 & LSDV & South Africa & (Lamien et al., 2011a) \\
\hline RPO30 & Niger-GU119952.1-LSDV & Cattle & GU119952.1 & LSDV & Niger & (Lamien et al., 2011a) \\
\hline RPO30 & South Africa-GU119948.1-LSDV & Springbok & GU119948.1 & LSDV & South Africa & (Lamien et al., 2011a) \\
\hline RPO30 & Burkina Faso-GU119946.1-LSDV & Cattle & GU119946.1 & LSDV & Burkina Faso & (Lamien et al., 2011a) \\
\hline RPO30 & South Africa-GU119943.1-LSDV & Cattle & GU119943.1 & LSDV & South Africa & (Lamien et al., 2011a) \\
\hline RPO30 & Sudan-GU119944.1-LSDV & Cattle & GU119944.1 & LSDV & Sudan & (Lamien et al., 2011a) \\
\hline RPO30 & South Africa-GU119937.1-LSDV & Cattle & GU119937.1 & LSDV & South Africa & (Lamien et al., 2011a) \\
\hline RPO30 & Turkey-GU119916.1-SPPV & Sheep & GU119916.1 & SPPV & Turkey & (Lamien et al., 2011a) \\
\hline RPO30 & Senegal-GU119926.1-SPPV & Sheep & GU119926.1 & SPPV & Senegal & (Lamien et al., 2011a) \\
\hline RPO30 & China-JQ310671.1-SPPV & Sheep & JQ310671.1 & SPPV & China & (Zhou et al., 2012) \\
\hline RPO30 & China-JQ310673.1-SPPV & Sheep & JQ310673.1 & SPPV & China & (Zhou et al., 2012) \\
\hline RPO30 & China-JQ310670.1-SPPV & Sheep & JQ310670.1 & SPPV & China & (Zhou et al., 2012) \\
\hline RPO30 & Algeria-GU119920.1-SPPV & Sheep & GU119920.1 & SPPV & Algeria & (Lamien et al., 2011a) \\
\hline RPO30 & Nigeria-GU119924.1-SPPV & Sheep & GU119924.1 & SPPV & Nigeria & (Lamien et al., 2011a) \\
\hline RPO30 & Turkey-GU119923.1-SPPV & Sheep & GU119923.1 & SPPV & Turkey & (Lamien et al., 2011a) \\
\hline RPO30 & Algeria-GU119921.1-SPPV & Sheep & GU119921.1 & SPPV & Algeria & (Lamien et al., 2011a) \\
\hline RPO30 & Turkey-GU119919.1-SPPV & Sheep & GU119919.1 & SPPV & Turkey & (Lamien et al., 2011a) \\
\hline RPO30 & Turkey-GU119918.1-SPPV & Sheep & GU119918.1 & SPPV & Turkey & (Lamien et al., 2011a) \\
\hline RPO30 & Turkey-GU119917.1-SPPV & Sheep & GU119917.1 & SPPV & Turkey & (Lamien et al., 2011a) \\
\hline RPO30 & Niger-GU119922.1-SPPV & Sheep & GU119922.1 & SPPV & Niger & (Lamien et al., 2011a) \\
\hline RPO30 & China-JQ310669.1-GTPV & Goat & JQ310669.1 & GTPV & China & (Zhou et al., 2012) \\
\hline
\end{tabular}

NA, not available. 

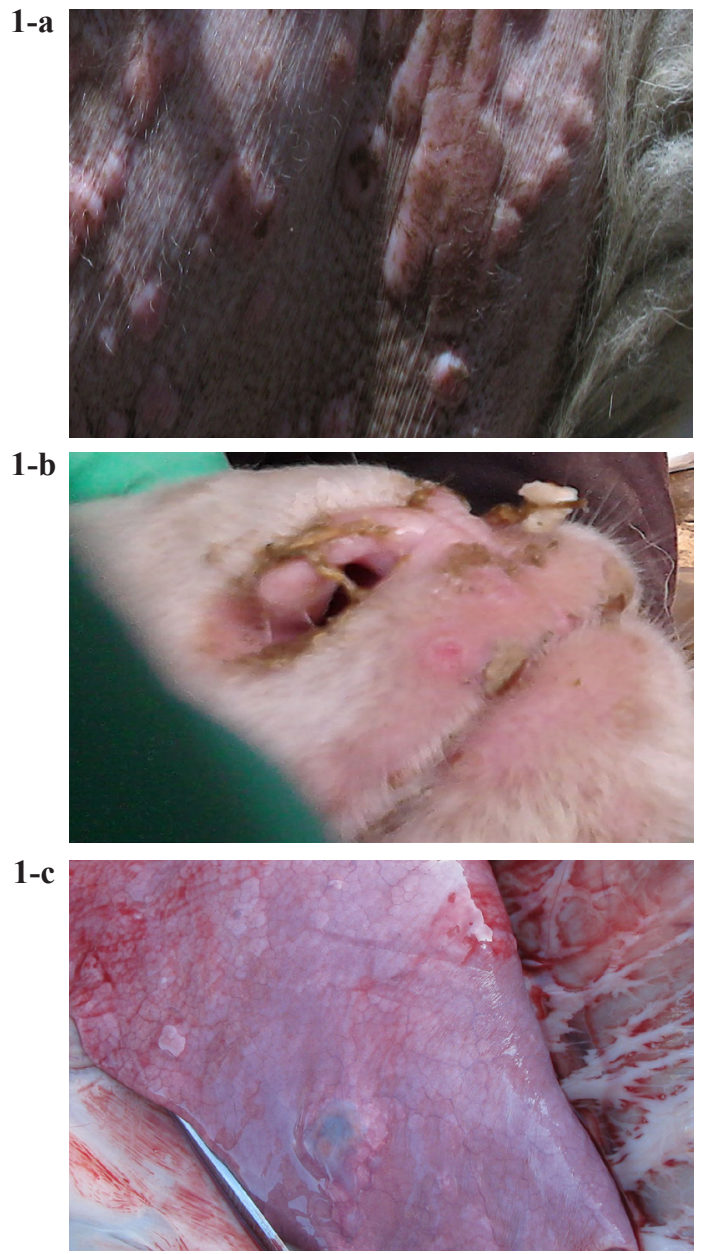

Figure 1. Representative clinical cases in Huining sheep flocks. 1-a. papules under the tail; 1-b. scabs on the lips; 1-c. pox in the liver.

Table 2. Percent identities of the $P 32$ genes of Huining and Zhangye isolates with other members of the genus Capripoxvirus.

\begin{tabular}{lcccc}
\hline & \multicolumn{2}{c}{ Huining isolate } & \multicolumn{2}{c}{ Zhangye isolate } \\
\cline { 2 - 3 } & Nucleotide & Amino acid & Nucleotide & Amino acid \\
\hline China isolates of capripoxvirus & $98.0-99.9$ & $96.9-99.7$ & $97.9-99.8$ & $96.3-99.4$ \\
China isolates of SPPV & $99.1-99.8$ & $97.2-99.4$ & $99.0-99.7$ & $96.9-99.4$ \\
China isolates of GTPV & $98.0-98.5$ & $96.6-97.5$ & $97.9-98.3$ & $96.6-97.2$ \\
Asia isolates of capripoxvirus & $98.0-99.9$ & $72.3-99.7$ & $97.9-99.8$ & $72.3-99.7$ \\
Asia isolates of SPPV & $98.0-99.9$ & $72.3-99.7$ & $97.9-99.8$ & $72.3-99.7$ \\
Asia isolates of GTPV & $98.0-98.5$ & $96.3-97.5$ & $97.9-98.3$ & $72.3-97.5$ \\
Worldwide isolates of capripoxvirus & $98.0-99.9$ & $72.3-99.7$ & $97.9-99.8$ & $72.3-99.7$ \\
Worldwide isolates of SPPV & $99.1-99.9$ & $72.3-99.7$ & $97.9-99.8$ & $96.6-97.2$ \\
Worldwide isolates of GTPV & $98.0-98.5$ & $96.6-97.5$ & $97.9-98.3$ & 99.7 \\
LSDV & $98.0-99.8$ & $72.3-97.5$ & 99.9 & 100 \\
Huining isolate & 100 & 99.7 & 97.7 \\
Zhangye isolate & 99.9 & & 100 \\
\hline
\end{tabular}


Table 3. Percent identities of the GPCR genes of Huining and Zhangye isolates with other members of the genus Capripoxvirus.

\begin{tabular}{|c|c|c|c|c|}
\hline & \multicolumn{2}{|c|}{ Huining isolate } & \multicolumn{2}{|c|}{ Zhangye isolate } \\
\hline & Nucleotide & Amino acid & Nucleotide & Amino acid \\
\hline China isolates of capripoxvirus & $95.2-99.7$ & $93.9-99.5$ & $95.2-99.7$ & $93.6-99.2$ \\
\hline China isolates of SPPV & $99.5-99.7$ & $99.5-99.7$ & $99.5-99.7$ & $99.2-99.7$ \\
\hline China isolates of GTPV & $95.2-95.4$ & 93.9 & $95.2-95.4$ & 93.6 \\
\hline Asia isolates of capripoxvirus & $95.2-99.7$ & $93.3-100$ & $95.2-99.7$ & $93.0-99.7$ \\
\hline Asia isolates of SPPV & $99.5-99.7$ & $99.5-100$ & $99.5-99.7$ & $99.2-100$ \\
\hline Asia isolates of GTPV & $95.2-99.6$ & $93.3-99.5$ & $95.2-99.6$ & $93.0-99.2$ \\
\hline Worldwide isolates of capripoxvirus & $95.0-99.7$ & $93.3-100$ & $95.0-99.7$ & $93.5-99.7$ \\
\hline Worldwide isolates of SPPV & $99.5-99.7$ & $99.2-100$ & $99.5-99.7$ & $98.9-99.7$ \\
\hline Worldwide isolates of GTPV & $95.2-99.6$ & $93.9-99.5$ & $95.2-99.6$ & $93.0-99.2$ \\
\hline LSDV & $95.0-95.7$ & $93.8-94.7$ & $95.0-95.7$ & $93.5-94.4$ \\
\hline Huining isolate & 100 & 100 & 99.8 & 99.7 \\
\hline Zhangye isolate & 99.8 & 99.7 & 100 & 100 \\
\hline
\end{tabular}

Table 4. Percent identities of the RPO30 genes of Huining and Zhangye isolates with other members of the genus Capripoxvirus.

\begin{tabular}{lcccc}
\hline & \multicolumn{2}{c}{ Huining isolate } & \multicolumn{2}{c}{ Zhangye isolate } \\
\cline { 2 - 3 } & Nucleotide & Amino acid & Nucleotide & Amino acid \\
\hline China isolates of capripoxvirus & $97.1-100$ & $98.5-100$ & $97.1-100$ & $98.5-100$ \\
China isolates of SPPV & $99.8-100$ & $99.5-100$ & $99.8-100$ & $99.5-100$ \\
China isolates of GTPV & $96.9-97.1$ & 98.5 & $96.9-97.1$ & 98.5 \\
Asia isolates of capripoxvirus & $96.4-100$ & $97.4-100$ & $96.4-100$ & $97.4-100$ \\
Asia isolates of SPPV & $99.8-100$ & $99.5-100$ & $99.8-100$ & $99.5-100$ \\
Asia isolates of GTPV & $96.4-97.1$ & $97.4-98.5$ & $96.4-97.1$ & $97.4-98.5$ \\
Worldwide isolates of capripoxvirus & $96.2-100$ & $97.4-100$ & $96.2-100$ & $97.4-100$ \\
Worldwide isolates of SPPV & $99.5-100$ & $99.5-100$ & $99.5-100$ & $97.5-100$ \\
Worldwide isolates of GTPV & $96.4-99.5$ & $97.4-99.5$ & $96.4-99.5$ & $97.4-99.5$ \\
LSDV & $96.2-96.6$ & $97.4-98$ & $96.2-96.6$ & 100 \\
Huining isolate & 100 & 100 & 100 & 100 \\
Zhangye isolate & 100 & & & 100 \\
\hline
\end{tabular}

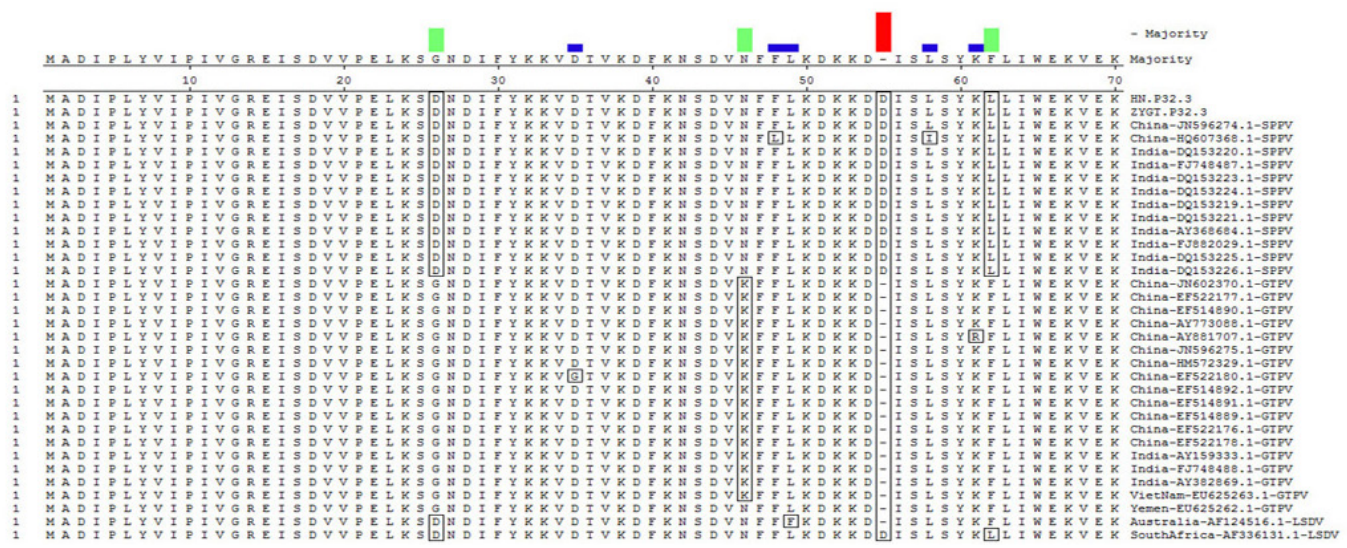

Figure 2. Alignment of the partial amino acid sequences of $P 32$ genes from several SPPV and GTPV strains. HN: Huining isolate; ZYGT: Zhangye isolate. 


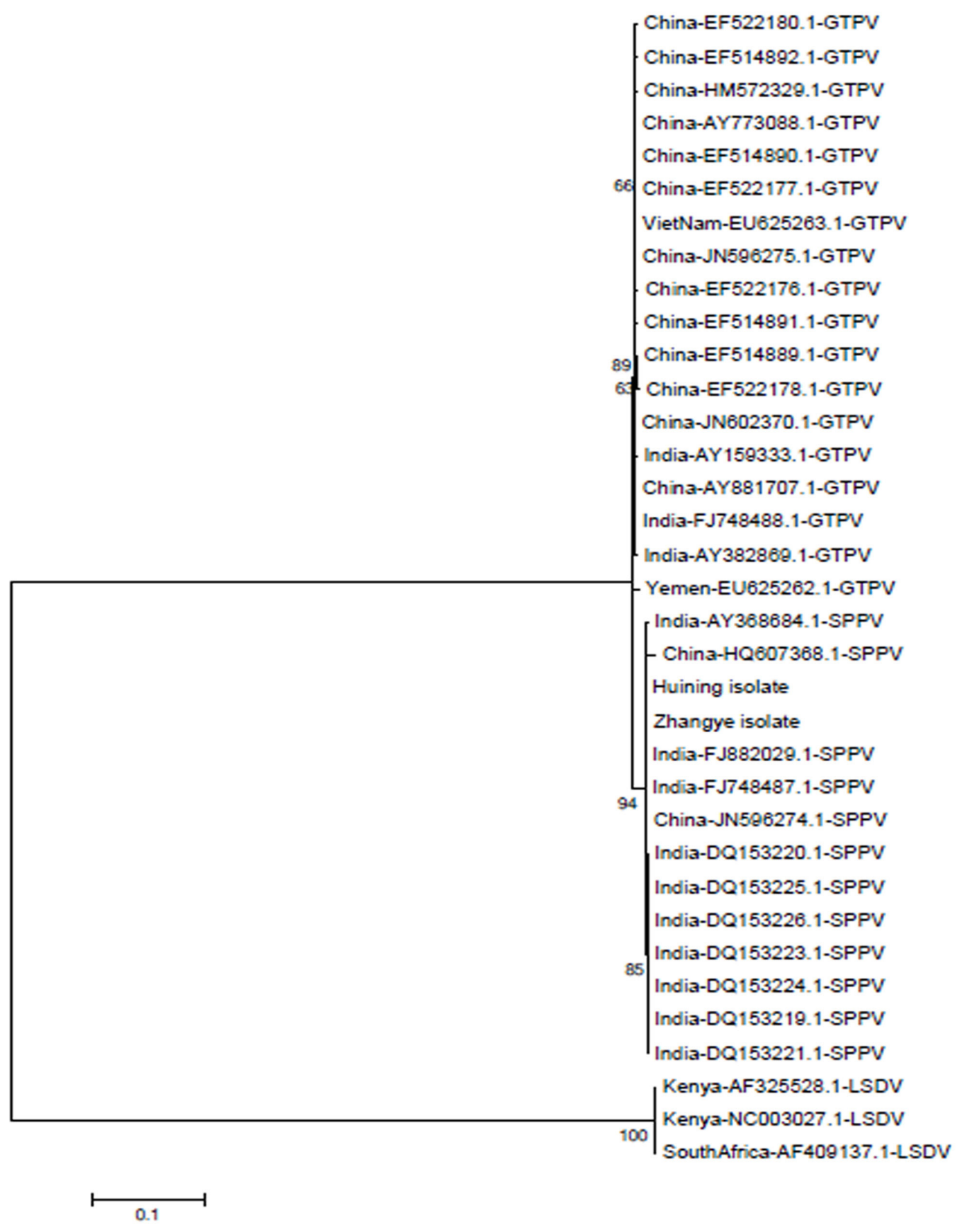

Figure 3. Phylogenetic analysis of different capripoxviruses based on the nucleotide sequences of the $P 32$ genes. 


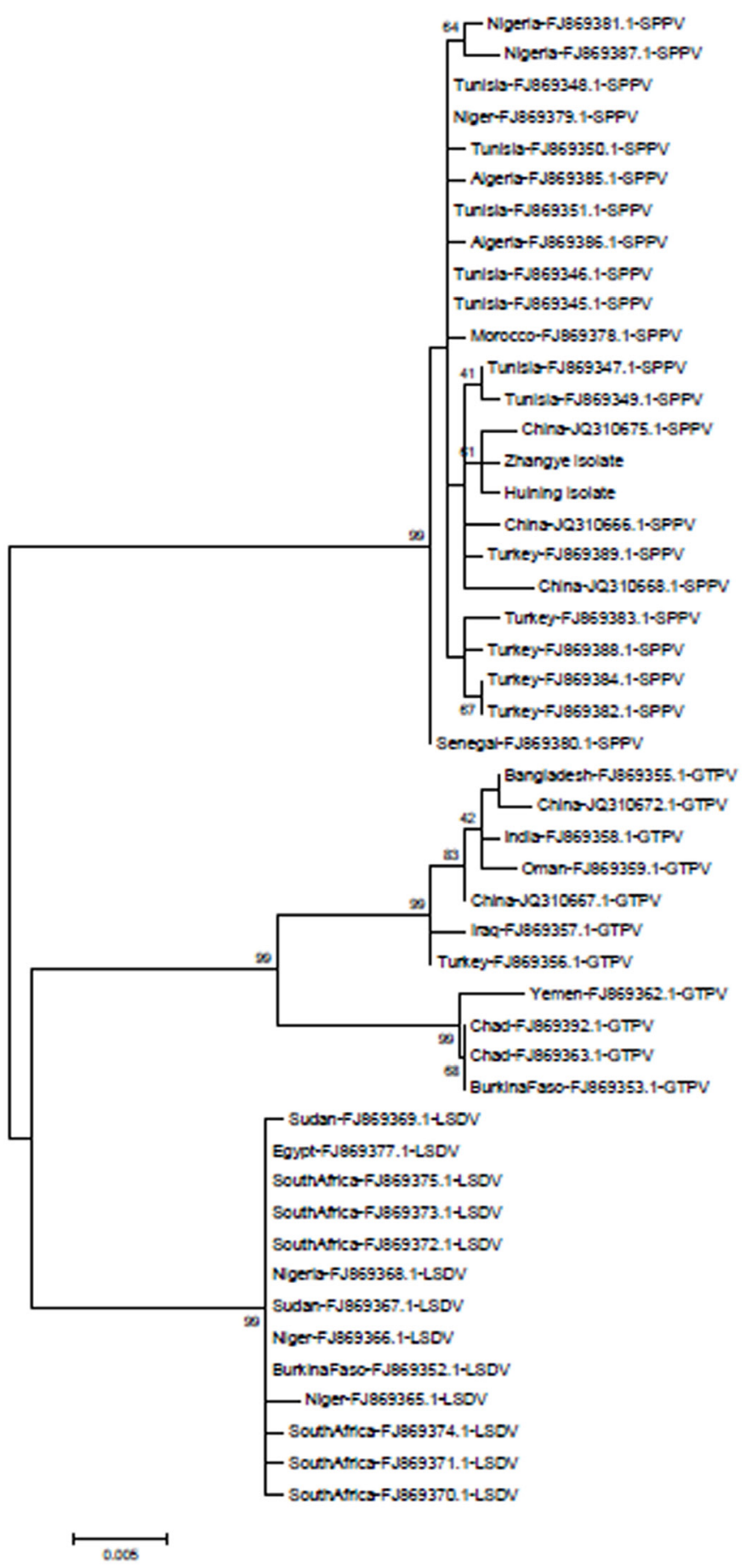

Figure 4. Phylogenetic analysis of different capripoxviruses based on the nucleotide sequences of the GPCR genes. 


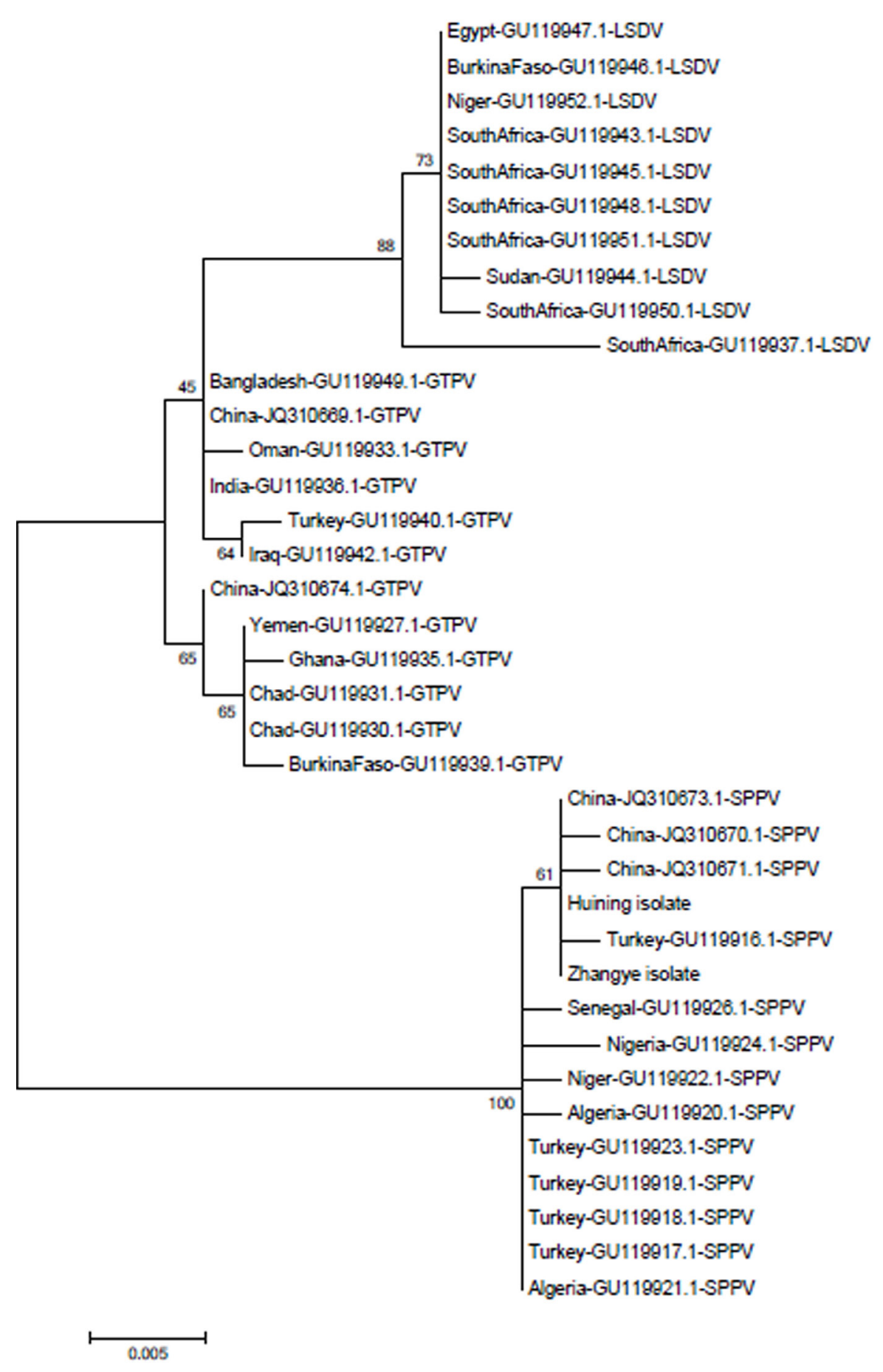

Figure 5. Phylogenetic analysis of different capripoxviruses based on the nucleotide sequences of the RPO30 genes. 
Based on the phylogenetic analysis of the $P 32, G P C R$, and RPO30 genes, the 2 strains were segregated into the SPPV group consistently in the 3 phylogenetic trees. There is a close relationship between the 2 isolates and Chinese SPPVs as they are clustered together. Because these ill sheep were imported from Jingyuan, another county of Gansu Province, our study strongly suggests the importance of veterinary surveillance prior to transportation.

\section{ACKNOLWEDGMENTS} (\#31260609).

Research sponsored by the National Natural Science Foundation to Yixia Chen

\section{REFERENCES}

Afshar A, Bundza A, Myers DJ, Dulac GC, et al. (1986). Sheep pox: experimental studies with a west African isolate. Can. Vet. J. 27: 301-306.

Ahn BY, Gershon PD, Jones EV and Moss B (1990). Identification of rpo30, a vaccinia virus RNA polymerase gene with structural similarity to a eucaryotic transcription elongation factor. Mol. Cell. Biol. 10: 5433-5441.

Asagba MO and Nawathe DR (1981). Evidence of sheep pox in Nigeria. Trop. Anim. Health Prod. 13: 61.

Babiuk S, Bowden TR, Parkyn G, Dalman B, et al. (2009). Yemen and Vietnam capripoxviruses demonstrate a distinct host preference for goats compared with sheep. J. Gen. Virol. 90: 105-114.

Beard PM, Sugar S, Bazarragchaa E, Gerelmaa U, et al. (2010). A description of two outbreaks of capripoxvirus disease in Mongolia. Vet. Microbiol. 142: 427-431.

Bhanuprakash V, Indrani BK, Hosamani M and Singh RK (2006). The current status of sheep pox disease. Comp. Immunol. Microbiol. Infect. Dis. 29: 27-60.

Bhanuprakash V, Venkatesan G, Balamurugan V, Hosamani M, et al. (2010). Pox outbreaks in sheep and goats at Makhdoom (Uttar Pradesh), India: evidence of sheeppox virus infection in goats. Transbound. Emerg. Dis. 57: 375-382.

Chakraborty C, George Priya Doss C, Sharma R, Sahana S, et al. (2013). Does computational biology help us to understand the molecular phylogenetics and evolution of cluster of differentiation (CD) proteins? Protein J. 32: 143-154.

Chensue SW (2001). Molecular machinations: chemokine signals in host-pathogen interactions. Clin. Microbiol. Rev. 14: 821-835, table of contents.

Embury-Hyatt C, Babiuk S, Manning L, Ganske S, et al. (2012). Pathology and viral antigen distribution following experimental infection of sheep and goats with capripoxvirus. J. Comp. Pathol. 146: 106-115.

Fields BN, Knipe DM and Howley PM (2007). Fields virology. Wolters Kluwer Health/Lippincott Williams \& Wilkins, Philadelphia.

Gulbahar MY, Davis WC, Yuksel H and Cabalar M (2006). Immunohistochemical evaluation of inflammatory infiltrate in the skin and lung of lambs naturally infected with sheeppox virus. Vet. Pathol. 43: 67-75.

Heine HG, Stevens MP, Foord AJ and Boyle DB (1999). A capripoxvirus detection PCR and antibody ELISA based on the major antigen $\mathrm{P} 32$, the homolog of the vaccinia virus H3L gene. J. Immunol. Methods 227: 187-196.

Hosamani M, Mondal B, Tembhurne PA, Bandyopadhyay SK, et al. (2004). Differentiation of sheep pox and goat poxviruses by sequence analysis and PCR-RFLP of P32 gene. Virus Genes 29: 73-80.

Johnson GP, Goebel SJ and Paoletti E (1993). An update on the vaccinia virus genome. Virology 196: 381-401.

Kara PD, Afonso CL, Wallace DB, Kutish GF, et al. (2003). Comparative sequence analysis of the South African vaccine strain and two virulent field isolates of Lumpy skin disease virus. Arch. Virol. 148: 1335-1356.

Lamien CE, Le Goff C, Silber R, Wallace DB, et al. (2011a). Use of the Capripoxvirus homologue of Vaccinia virus 30 kDa RNA polymerase subunit (RPO30) gene as a novel diagnostic and genotyping target: development of a classical PCR method to differentiate Goat poxvirus from Sheep poxvirus. Vet. Microbiol. 149: 30-39.

Lamien CE, Lelenta M, Goger W, Silber R, et al. (2011b). Real time PCR method for simultaneous detection, quantitation and differentiation of capripoxviruses. J. Virol. Methods 171: 134-140.

Le Goff C, Lamien CE, Fakhfakh E, Chadeyras A, et al. (2009). Capripoxvirus G-protein-coupled chemokine receptor: a host-range gene suitable for virus animal origin discrimination. J. Gen. Virol. 90: 1967-1977.

McCormack GP and Clewley JP (2002). The application of molecular phylogenetics to the analysis of viral genome diversity and evolution. Rev. Med. Virol. 12: 221-238. 
Oğuzoğlu TC, Alkan F, Ozkul A, Vural SA, et al. (2006). A sheeppox virus outbreak in Central Turkey in 2003: isolation and identification of capripoxvirus ovis. Vet. Res. Commun. 30: 965-971.

Rao TV and Bandyopadhyay SK (2000). A comprehensive review of goat pox and sheep pox and their diagnosis. Anim. Health Res. Rev. 1: 127-136.

Roy P, Purushothaman V, Sreekumar C, Tamizharasan S, et al. (2008). Sheep pox disease outbreaks in Madras Red and Mechery breeds of indigenous sheep in Tamilnadu, India. Res. Vet. Sci. 85: 617-621.

Sanderson MJ and Wojciechowski MF (2000). Improved bootstrap confidence limits in large-scale phylogenies, with an example from Neo-Astragalus (Leguminosae). Syst. Biol. 49: 671-685.

Seet BT and McFadden G (2002). Viral chemokine-binding proteins. J. Leukoc. Biol. 72: 24-34.

Tian H, Chen Y, Wu J, Shang Y, et al. (2010). Serodiagnosis of sheeppox and goatpox using an indirect ELISA based on synthetic peptide targeting for the major antigen P32. Virol. J. 7: 245.

Tulman ER, Afonso CL, Lu Z, Zsak L, et al. (2001). Genome of lumpy skin disease virus. J. Virol. 75: 7122-7130.

Tulman ER, Afonso CL, Lu Z, Zsak L, et al. (2002). The genomes of sheeppox and goatpox viruses. J. Virol. 76: 60546061.

Zhou T, Jia H, Chen G, He X, et al. (2012). Phylogenetic analysis of Chinese sheeppox and goatpox virus isolates. Virol. J. 9: 25. 